\title{
Laser Based Real-Time Measurement of Thorax 3D Deformation with Motion Compensation
}

\author{
Klemen POVŠIČ, Janez MOŽINA, Matija JEZERŠEK \\ University of Ljubljana, Faculty of Mechanical Engineering, Ljubljana, Slovenia
}

\begin{abstract}
We present a novel method for full-area, 3D visual breathing assistance with body motion compensation. The method is based on Kinect structured light triangulation sensor and uses local features detection algorithm to correct the undesired body motion during breathing training. The measuring system is contactless and operates in real-time with 30 frames per second. The feature detection algorithm is based on finding the best match between the reference template and the acquired texture image. This is performed by calculating the normalized cross-correlation inside the specified region of interest for four markers attached to the patient's thoraco-abdominal region. The motion compensation is achieved by $3 \mathrm{D}$ transformation of the acquired data with respect to the spatial coordinates of the detected markers. This enables the monitoring of respiratory-related displacements only, disregarding other displacements incurred by body motion.

The displacements of the current surface with respect to the reference surface are displayed using a color gradient, which offers visual support to the patients and helps to control respiratory motion according to the training requirements. The results of the study show that the 3D measuring system with motion compensation is robust and provides a reliable and unbiased method of breathing pattern assessment.
\end{abstract}

Keywords: laser measurement, 3D, real-time, thorax, body motion, respiration

\section{Introduction}

Respiration monitoring systems in medicine have been developed to offer a reliable and quantitative method to assess breathing dynamics and to diagnose or correct breathing disorders. Such measuring systems can be contact or non-contact and are used in numerous clinical settings $[1,2,3$, 4]. Contact measuring methods rely on direct physical contact with the measured subject. These conventional systems which are mainly based on spirometers [5], magnetometers [1], stretching belts [6], plethysmographs [7], optical fibers [8] and electrocardiograms [9] can interfere with the breathing pattern and consequently increase measurement errors. Such methods also lack the possibility of evaluating the entire region deformation. Contrarily, due to the noninvasive principle of measurements the non-contact measuring systems do not interfere with the breathing or cause any additional discomfort to the patient. One method for determining the respiration rate is monitoring the skin temperature profile associated with breathing using an infrared thermal camera [10]. Another method is single camera photogrammetry, where different approaches of monitoring the chest wall displacements are used [4, 11]. Majority of optical measuring systems are based on stereophotogrammetry [12, 13, 14], optoelectronic plethysmography [15, 16], laser triangulation [17, 18], color structured light triangulation [19], laser vibrometry [20], Fourier profilometry [21] and Timeof-Flight (TOF) [22]. Mizobe et al. presented a system for pulmonary function testing using a dot matrix pattern projector and a CCD camera [23]. They examined the relationship between the flow volume and the volume change of the thorax surface. Jezeršek et al. improved this method by simultaneously using multiple measuring modules [24]. This was achieved with the color modulation principle. Recently, similar method for tidal volume evaluation and breathing pattern assessment was used by Povšič et al. [25]. They presented a full-area 3D visual feedback method for teaching and correcting breathing.

The goal of the developed system is to enable real-time, full-area 3D visual breathing assistance to patients with chronic obstructive pulmonary disease (COPD). Such patients are clinically trained to increase the breathing capacity. The visualization of active thorax regions can improve the training results. In order to measure only the respiratory related displacements, the body motion is eliminated. Verification experiments are performed in order to determine the system's capabilities. 


\section{Measuring setup}

Figure 1 shows the measurement configuration, where the depth and texture information of the patient's chest wall is acquired by the measuring system. In the next step, the signal is processed using a personal computer and the 3D shape is displayed on the monitor with the purpose of visual support during the breathing exercise in real-time.

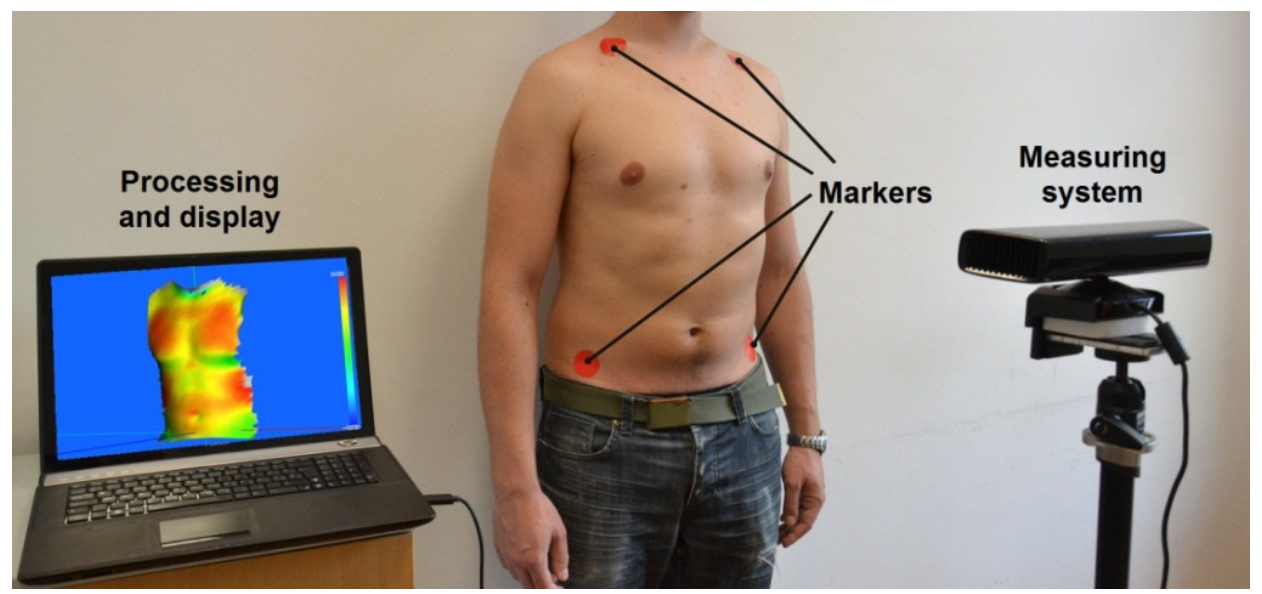

Fig. 1. Measurement configuration.

The schematic diagram of the measuring system is shown in Figure 2. The Kinect sensor uses a structured light pattern that is projected on the patient's torso and acquired with the near infrared (NIR) camera [26]. Simultaneously, the texture is also detected using a color (RGB) camera. Since the principal axis of the depth and the texture image are misaligned, this has to be taken into account during the calibration/processing stage. After the images are acquired, post-processing is performed to display the reconstructed 3D shape and visualize the deformations using the color gradient.

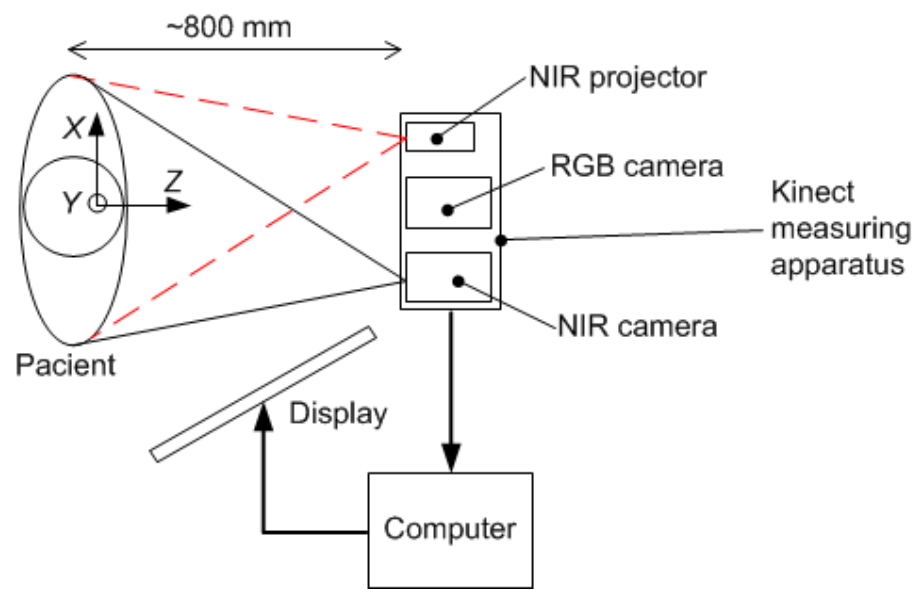

Fig. 2. Schematic diagram of the measuring system.

The measuring system was placed approximately $800 \mathrm{~mm}$ away from the patient in such a way that it covered the entire thoraco-abdominal region. The measuring range of Kinect is $900 \times 700 \times 600 \mathrm{~mm}$ (width, height and depth) at this distance and resolution is approximately $1.7 \mathrm{~mm}$ [26]. Four passive circular red markers were placed on the measured surface as shown in Figure 1. Their position was determined by observing the least active regions, i.e. the regions where the displacements induced by breathing along the Z-axis are minimal. Considering this, two markers were symmetrically placed on the patient's clavicle and the other two also symmetrically on the ilium. Markers were simultaneously tracked and thus the body motion was actively compensated throughout the breathing experiments.

To successfully detect the placed markers during the measurements, it is important to ensure the best contrast possible. This is achieved by performing the detection on the green image plane, where the markers are clearly visible and distinct from the background. 


\section{Real-time measuring process}

The system operates in real-time according to the data processing diagram shown in Figure 3 . Using the Kinect Primesense drivers, OpenNI and PCL library [27, 28, 29], the 3D data is expressed in world coordinates and simultaneously aligned with the acquired texture information. The entire measuring process consists of three parts: acquisition, processing and visualization.

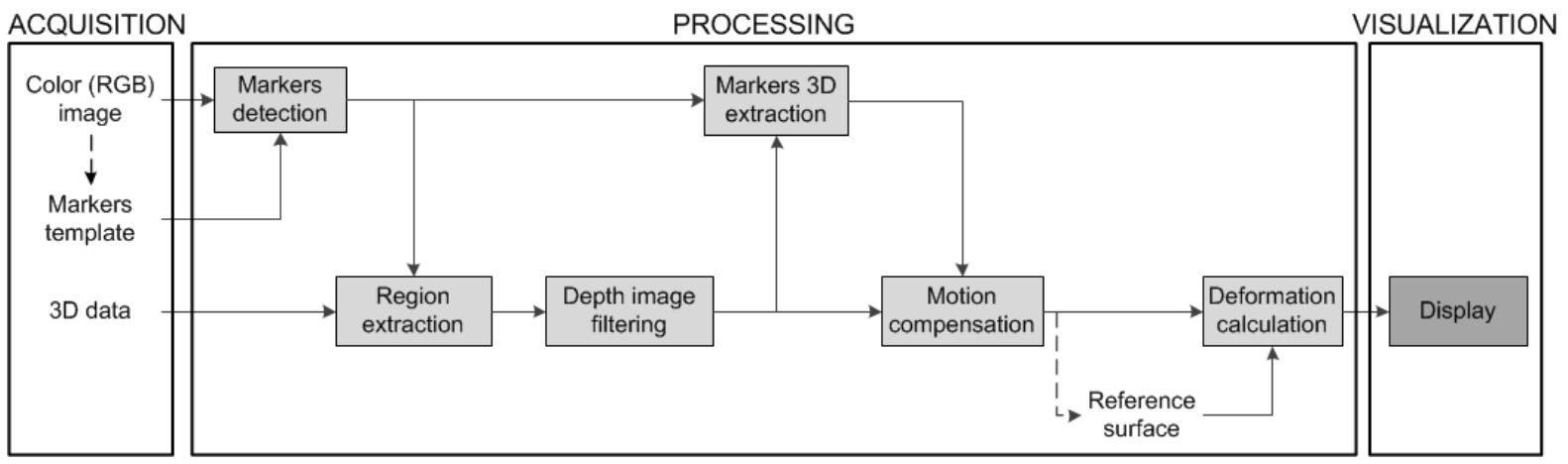

Fig. 3. Real-time data processing diagram.

First, the depth and the color images are acquired by Kinect and the templates of the markers are stored in the buffer memory. Next, the templates are moved inside the respective region of interest $(\mathrm{ROI})$ on the color image and the best matches are found using the cross-correlation algorithm with sub-pixel resolution (see Figure 4). The detection inside a specified ROI significantly speeds up the entire process as the average match time was found to be approximately 1 millisecond for all markers. Afterwards, the markers ROI location is automatically adapted in the image so that the center coincides with the center of the matched marker template. The acquired depth and color image are aligned, which enables to perform the markers 3D location extraction, where the location of each individual marker in world coordinate system is known. The detected markers form the measuring region that is used for further processing.

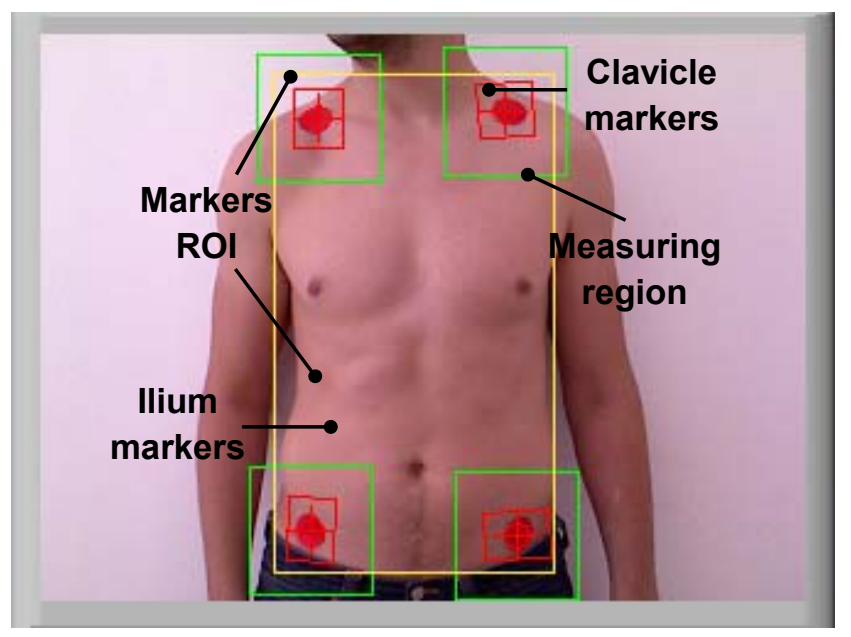

Fig. 4. Detection region of interest (ROI) and measuring area on the patient's chest wall.

In the next step, a filter is applied to the depth coordinates, which removes the noise and consequently smooth the surface. Next, the measurement is compensated by applying translation and rotation to the acquired data in 3D space with respect to the spatial coordinate points of the detected markers. The motion compensation consists of five sub-steps (see Figure 5 and Figure 6). 
(a)

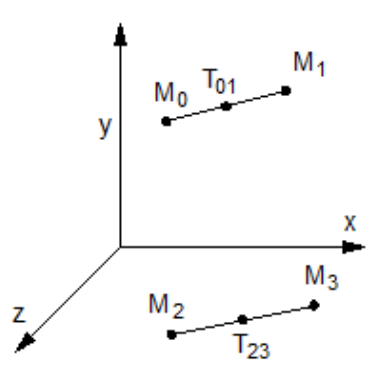

(c)

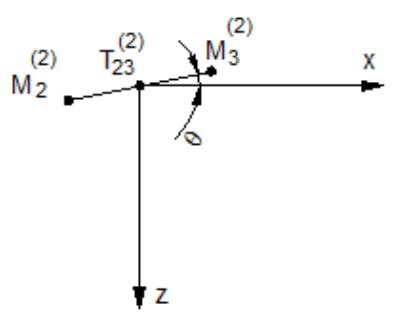

(1) (b)

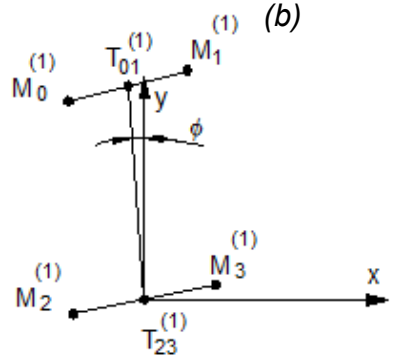

(d)

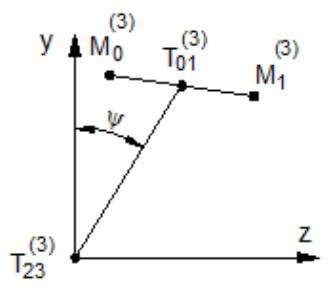

Fig. 5.3D motion compensation. $M_{0}, M_{1}$ represent the clavicle markers and $M_{2}, M_{3}$ the ilium markers in the initial position (a). First, the translation to the coordinate system origin is performed (b), followed by three rotations around $Z, Y$ and $X$ axes respectively $(b-d)$.

First, the origin of the world coordinates is translated in the middle $\left(T_{23}\right)$ between the ilium points $\left(M_{2}\right.$ and $\left.\mathrm{M}_{3}\right)$. Then, the rotation around $Z$-axis by angle $\phi$ is followed so that the middle $\left(T_{01}{ }^{(1)}\right)$ between the clavicle points $\left(M_{0}\right.$ and $\left.M_{1}\right)$ lies on $Y$-axis. Next, the rotation around $Y$-axis by angle $\theta$ aligns the ilium points with the $\mathrm{X}$-axis. Another rotation around the $\mathrm{X}$-axis by angle $\psi$ is performed to align the middle $\left(\mathrm{T}_{01}{ }^{(3)}\right)$ of the clavicle markers with the Y-axis. Lastly, the torsional rotation along the vertical body axis ( $Y$-axis) is undistorted using equations 1 to 3 and assuming that the ilium markers are aligned with the $\mathrm{X}$-axis in the world coordinate system (see Figure 6b):

$$
\begin{aligned}
& x_{\text {und }}=x_{d} \cos (\theta(y))+z_{d} \sin (\theta(y)) \\
& z_{\text {und }}=-x_{d} \sin (\theta(y))+z_{d} \cos (\theta(y))
\end{aligned}
$$

where $x_{d}$ and $z_{d}$ are distorted coordinates while $x_{\text {und }}$ and $z_{\text {und }}$ are the respective undistorted coordinates. The variable $\theta(y)$ is calculated by the following equation:

$$
\theta(y)=\theta_{H} \cdot \frac{y}{H}
$$

The $\theta_{H}$ is the angle between the markers and the $\mathrm{X}$-axis and $H$ is the distance between the clavicle and the ilium markers along the $\mathrm{Y}$-axis.

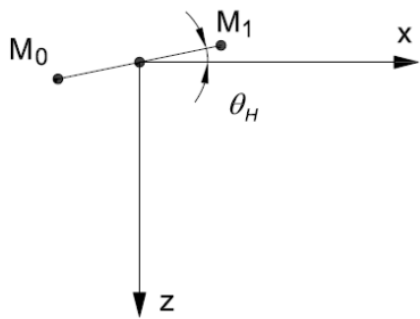

(a)

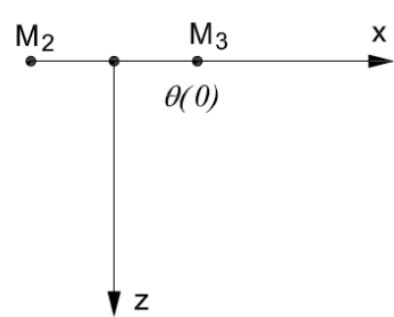

(b)

Fig. 6. Torsion deformation correction along vertical $y$-axis. $M_{0}$ and $M_{1}$ represent the clavicle markers, whereas $M_{2}$ and $M_{3}$ represent the ilium markers. The angle $\theta(y=H)$ is defined by the position of the markers (a). At $\theta(y=$ 0) the angle is equal to zero (b). 
In the final stage, the 3D surface reconstruction is performed, where the points in 3D space are triangulated and displayed on the monitor. The displacements from the selected reference surface are represented using a color gradient to visualize the most active body regions during breathing (see chapter 4.3).

\section{System verification}

System was first tested in order to determine the uncertainty of the markers detection and motion compensation algorithm. The same reference, i.e. static plain surface, was used in both tests to measure the average standard deviation of the markers position and the average depth deviation of the measured surface. The experiments were performed under normal indoor lighting conditions, where the iluminance is approximately $500 \mathrm{Ix}$.

\subsection{Uncertainty of markers detection}

To determine the marker detection uncertainty, four markers were placed on a flat surface as shown in Figure 7. The marker positions in the image coordinate system were tracked in real-time using subpixel accuracy.

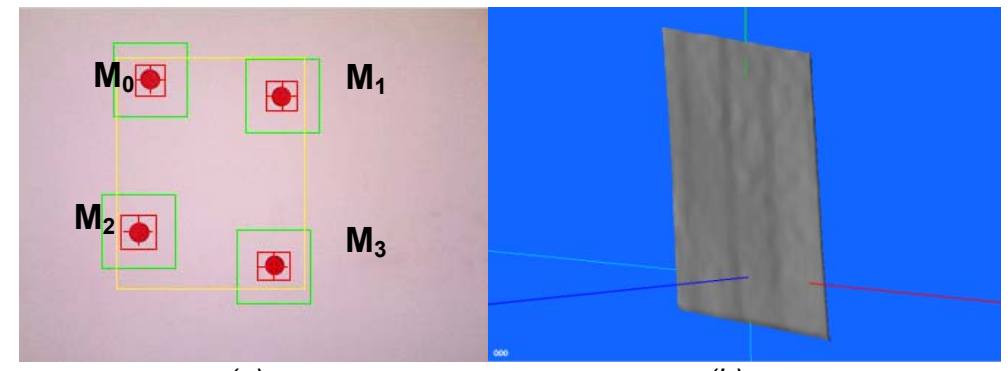

(a)

(b)

Fig. 7. Flat surface with markers (a) and reconstructed $3 D$ shape (b) used to determine the detection and transformation errors.

The standard deviation of each detected marker center $(u, v)$ in image pixels was calculated based on multiple data samples ( $n=1000$ samples) with the results shown in Table 1.

Table 1.Standard deviation for markers position detection in image coordinates $(u, v)$ for 1000 measured samples.

\begin{tabular}{|c|c|c|}
\cline { 2 - 3 } \multicolumn{1}{c|}{} & $\mathrm{u}$ [pix] & $\mathrm{v}$ [pix] \\
\hline $\mathrm{M}_{0}$ & 0.02 & 0.02 \\
\hline $\mathrm{M}_{1}$ & 0.01 & 0.02 \\
\hline $\mathrm{M}_{2}$ & 0.01 & 0.02 \\
\hline $\mathrm{M}_{3}$ & 0.02 & 0.02 \\
\hline
\end{tabular}

The average detection error of centers on the image plane for all markers was calculated and found to be \pm 0.017 pixels.

\subsection{Uncertainty of motion compensation}

The uncertainty of motion compensation was determined using the same reference surface as described in Section 4.1. In this case, the position of the measuring system was shifted from left to right and rotated around all three principle axes inside a specified range with respect to the initial position (see Figure 8 and Table 2). For each measured surface its average $Z$ value $\left(\bar{Z}_{i}^{\text {surf }}\right)$ was calculated. Standard deviation of these values was chosen as a measure for uncertainty (see equation 4).

where

$$
\sigma_{\mathrm{s}}=\sqrt{\frac{1}{n} \cdot \sum_{i=1}^{n}\left(\bar{Z}_{i}^{\text {surf }}-\mu\right)^{2}}
$$

$$
\mu=\frac{1}{n} \sum_{i=1}^{n} \bar{Z}_{i}^{\text {surf }}
$$




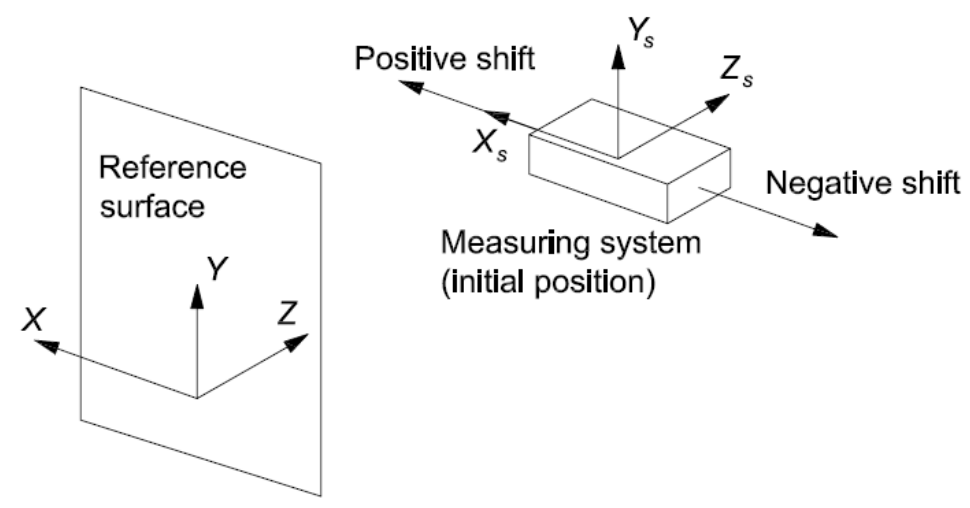

Fig. 8. Setup for determining the uncertainty of motion compensation. The measuring system was shifted along the $X_{s}$-axis in both directions and rotated around all three axes $\left(X_{s}, Y_{s}\right.$ and $\left.Z_{s}\right)$.

Table 2. Position changes of the measuring system with respect to its coordinate system.

\begin{tabular}{|c|c|}
\cline { 2 - 2 } \multicolumn{1}{c|}{} & Range \\
\hline Shift $[\mathrm{mm}]$ & \pm 150 \\
\hline Rotation around $X_{\mathrm{s}}$-axis [deg] & \pm 10 \\
\hline Rotation around $Y_{s}$-axis [deg] & \pm 10 \\
\hline Rotation around $Z_{s}$-axis [deg] & \pm 12 \\
\hline Uncertainty deviation $(\boldsymbol{n}=\mathbf{1 0 0 0}) \sigma_{\mathbf{s}}[\mathrm{mm}]$ & $\mathbf{\pm 1 . 2 7}$
\end{tabular}

The calculated uncertainty deviation for motion compensation based on 1000 data samples was found to be $\pm 1.27 \mathrm{~mm}$.

\subsection{Breathing measurement}

The deformation of the measured body was visually evaluated using the color representation as shown in Figure 9. In the initial stage, full exhalation was performed and the reference surface was saved. This was followed by full inhalation and breath-holding during the vertical rotation of the measured body to the right and to the left.

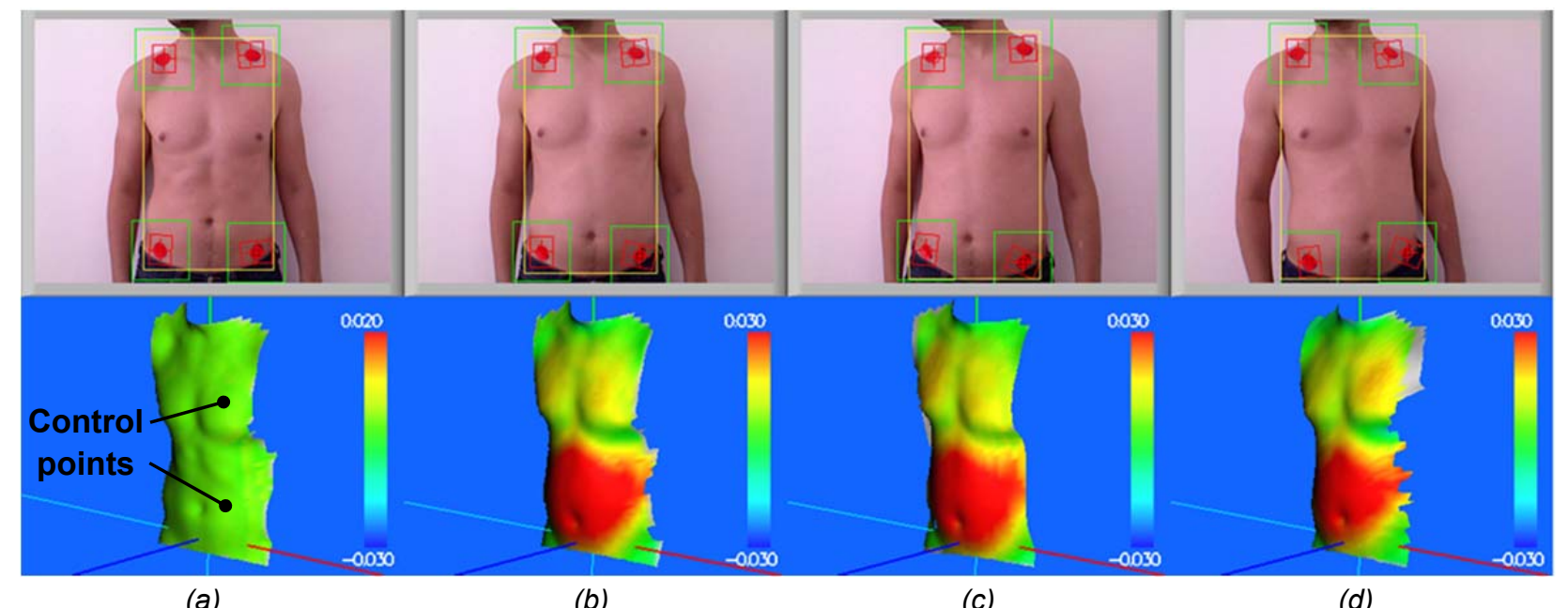

(a)

(b)

(c)

(d)

Figure 9. Sequence of thorax image (1st row) and $3 D$ shape (2nd row) showing the displacements using a color gradient. The test was performed with initial full exhalation, when the reference surface was saved (a); followed by full inhalation (b) and breath-holding during vertical right-rotation (c) and left-rotation (d).

The images in Figure 9 show that the body motion has no significant impact on the displacements related to breathing, since the active regions remain the same throughout the measurement. To further substantiate this, the relative depth of two selected control points on the 3D reconstructed surface was measured using the same sequence of acquired images (see Figure 9, second row). 
The measurements were performed with both disabled and enabled motion compensation algorithm and the depth of the corresponding points was compared. Ideally, the relative depth of both control points during breath-holding with motion compensation should be constant. However, this is impossible to achieve, since there are many factors that impact this; mainly the characteristics of the human respiratory mechanics and the accuracy of the measuring system.

Figure 10 shows the relative depth of the two control points for both methods (i.e. "motion compensation" and "no motion compensation"). At the beginning full inhalation is performed, followed by the breath-holding and body motion as previously described (see Figure 9 ).
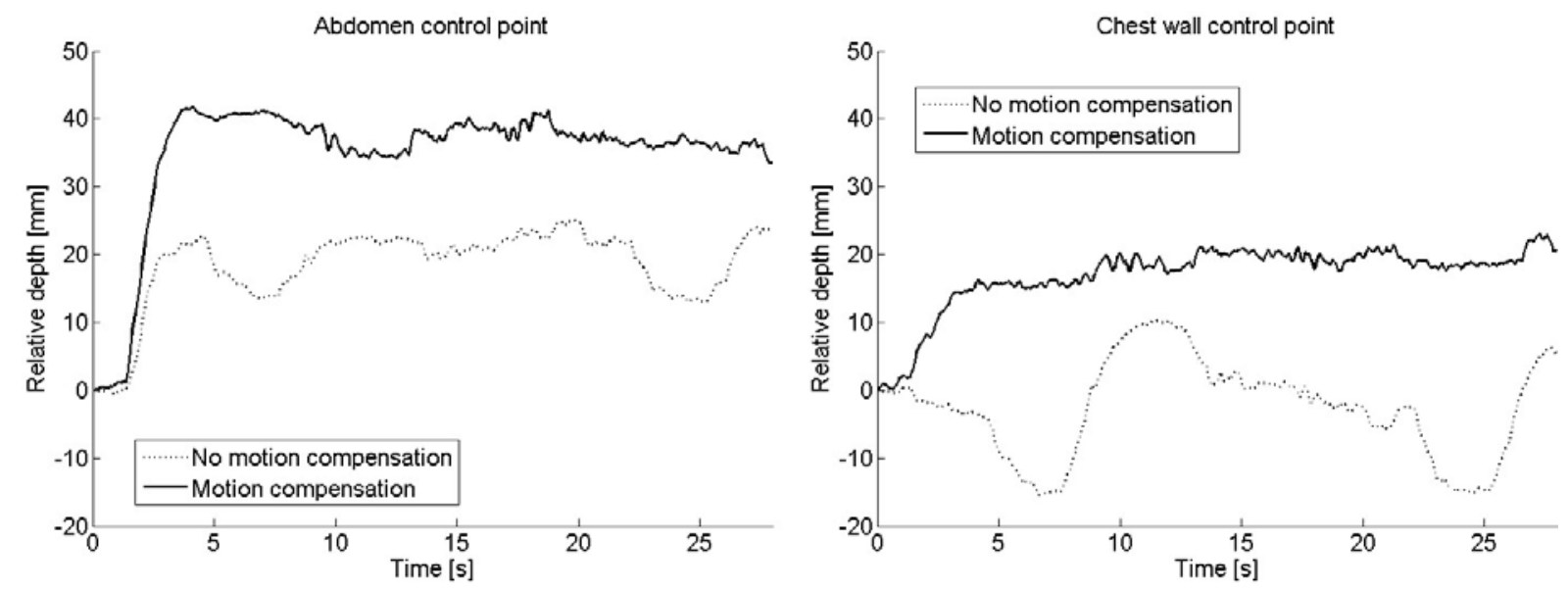

Figure 10. Control points on the $3 D$ reconstructed surface. The depth of both points, relative to the reference surface was measured throughout the measurement sequence.

In case of motion compensation, it is clearly visible that the body motion has significantly smaller impact on the relative depth change of both control points. The effect of the torsional deformation correction can also be seen by observing the depth changes of the chest wall control point, since its location lies between the clavicle and the ilium markers along the Y-axis.

\section{Conclusion}

The paper presents a full-area, real-time 3D laser system for thorax deformation measurement during breathing. The main novelty is the motion compensation algorithm. It consists of translating and rotating the acquired 3D data using the spatial coordinates of the four detected markers as reference points. The markers are placed on the patient's thoraco-abdominal surface and tracked throughout the measurements.

The purpose of the developed system is to offer unambiguous and simple visual feedback support to the patients during the breathing exercises. In this case, the body motion needs to be considered as it influences the measurements and consequently makes the visualization inaccurate. Experiments show that the proposed method uses a robust detection algorithm in combination with the 3D measuring system to effectively compensate the patient's body motion and displays only the deformations related to breathing.

\section{References}

1. Loring, S. H., Townsend, S. R., et al. (2006): "Expiratory Abdominal Rounding in Acute Dyspnea Suggests Congestive Heart Failure", Lung, Vol. 184, No.6, pp. 324-329.

2. Hart, A., Tallevi, K., et al. (2010): "A contact-free respiration monitor for smart bed and ambulatory monitoring applications", Conf. Proc. IEEE Eng. Med. Biol. Soc. 2010, Buenos Aires, pp. 927-930.

3. Wu, D., Wang L., et al. (2009): "A wearable respiration monitoring system based on digital respiratory inductive plethysmography", Conf. Proc. IEEE Eng. Med. Biol. Soc. 2009, Minneapolis, pp. 4844-4847.

4. Ricieri, D. D. and Rosario, N. A., (2009): "Effectiveness of a photogrammetric model for the analysis of thoracoabdominal respiratory mechanics in the assessment of isovolume maneuvers in children", Jornal Brasileiro De Pneumologia, Vol. 35, No. 2, pp. 144-150.

5. Arne, M., Lisspers, K., et al. (2010): "How often is diagnosis of COPD confirmed with spirometry?", Respiratory Medicine, Vol. 104, No. 4, pp. 550-556. 
6. Shaopeng, L., Gao, R. X., et al. (2011): "Non-invasive respiration and ventilation prediction using a single abdominal sensor belt", Signal Processing in Medicine and Biology Symposium 2011, Brooklyn, pp. 1-5.

7. Grossman, P., Wilhelm, F. H., et al. (2010): "Accuracy of ventilatory measurement employing ambulatory inductive plethysmography during tasks of everyday life", Biological Psychology, Vol. 84, No. 1, pp. 121-128.

8. Augousti, A. T., Maletras, F. X. and Mason, J., (2005): "Improved fibre optic respiratory monitoring using a figure-of-eight coil", Physiol. Meas., Vol. 26, No. 5, pp. 585-590.

9. Park, S. B., Y. S. Noh, et al. (2008). "An improved algorithm for respiration signal extraction from electrocardiogram measured by conductive textile electrodes using instantaneous frequency estimation." Med. Biol. Eng. Comput. 46(2): 147-158.

10. Abbas, A. K., Heiman, K., et al. (2010): "Non-Contact Respiratory Monitoring Based on Real-Time IR-Thermography", World Congress on Medical Physics and Biomedical Engineering 2009, Munich, pp. 1306-1309.

11. Tan, K. S., Saatchi, R., et al. (2010): "Real-time vision based respiration monitoring system", 7th International Symposium on Communication Systems Networks and Digital Signal Processing 2010, Newcastle upon Tyne, pp. 770 - 774.

12. Beach, R. D., Pretorius, P. H., et al. (2004): "Feasibility of Stereo-Infrared Tracking to Monitor Patient Motion During Cardiac SPECT Imaging", IEEE Trans. Nucl. Sci., Vol. 51, No. 5, pp. 26932698.

13. Grotte, A. D., et al. (1997): "Chest wall motion during tidal breathing", J. Appl. Physiol., Vol. 83, No. 5, pp. 1531-1537.

14. Beach, R. D., Gifford, H. C., et al. (2005): "Stereo-infrared tracking to monitor and characterize rigid-body motion and respiration during cardiac SPECT imaging: progress towards robust clinical utilization", Nuclear Science Symposium Conference Record 2005, Puerto Rico, pp. 1731-1735.

15. Dellaca, R. L., Ventura, M. L., et al. (2010): "Measurement of Total and Compartmental Lung Volume Changes in Newborns by Optoelectronic Plethysmography", Pediatric Research, Vol. 67, No. 1, pp. 11-16.

16. Layton, A. M., Garber, C. E., et al. (2011): "An assessment of pulmonary function testing and ventilatory kinematics by optoelectronic plethysmography", Clin. Physiol. Funct. Imaging, Vol. 31, No. 5, pp. 333-336.

17. Kondo, T., et al. (2006): "Noninvasive monitoring of chest wall movement in infants using laser", Pediatr. Pulm., Vol. 41, No. 10, pp. 985-992.

18. Aoki, H., Koshiji, K., et al. (2005): "Study on respiration monitoring method using near-infrared multiple slit-lights projection", IEEE International Symposium on Micro-NanoMechatronics and Human Science 2005 , Nagoya, pp. 291-296.

19. Chen, H. J., et al., (2010): "Color structured light system of chest wall motion measurement for respiratory volume evaluation", J. Biomed. Opt., Vol. 15, No. 2, pp. 026013-1 - 026013-9.

20. Scalise, L., Ercoli, I., et al. (2011): "Measurement of respiration rate in preterm infants by laser Doppler vibrometry", International Workshop on Medical Measurements and Applications Proceedings 2011, Bari, pp. 657-661.

21. Price, G. J., Parkhurst, J. M., et al. (2012): "Real-time optical measurement of the dynamic body surface for use in guided radiotherapy", Physics in Medicine and Biology, Vol. 57, No. 2, pp. 415436.

22. Wentz, T., Fayad, H., et al. (2012), "Accuracy of dynamic patient surface monitoring using a timeof-flight camera and B-spline modeling for respiratory motion characterization", Phys. Med. Biol., Vol. 57, No. 13, pp. 4175-4193.

23. Mizobe, Y., Aoki, H. and Koshiji, K., (2007): "Basic study on relationship between respiratory flow volume and volume change of thorax surface", Proc. Int. Special Topic Conf. on Information Technology Applications in Biomedicine 2007, Tokyo, pp. 219-222.

24. Jezeršek, M. and Možina, J., (2009): "High-speed measurement of foot shape based on multiplelaser-plane triangulation", Opt. Eng., Vol. 48, No.11, pp. 113604-1 - 113604-8.

25. Povšič, K., Fležar, M., Možina, J. and Jezeršek, M., (2012): "Laser 3-D measuring system and real-time visual feedback for teaching and correcting breathing", J. Biomed. Opt., Vol. 17, No. 3, pp. 036004-1 - 036004-8.

26. Khoshelham, K. and Elberink, S.O, (2012): "Accuracy and resolution of Kinect depth data for indoor mapping applications", Sensors, Vol. 12, No. 2, pp. 1437-1454.

27. PrimeSense (accessed 2012): http://www.primesense.com.

28. OpenNI (accessed 2012): http://openni.org/.

29. PCL - Point Cloud Library (accessed 2012): http://pointclouds.org/. 